\title{
Bilateral Tubo-Ovarian Abscesses Four Years After Total Abdominal Hysterectomy
}

\author{
L.E. Mendez, S.M. Bhoola, and I.R. Horowitz* \\ Department of Gynecology and Obstetrics, Division of Gynecologic Oncology, and the Winship Cancer \\ Center, Emory University School of Medicine, Atlanta, GA
}

\begin{abstract}
Background: Pelvic inflammatory disease (PID) is a common gynecologic disorder. One known complication of PID is tubo-ovarian abscess (TOA) formation. The predominant theory on TOA formation postulates that an ascending infection from the cervix through the uterus to the fallopian tubes and ovaries results in abscess formation. Other theories include seeding via a hematogenous infection, diverticular disease, and appendicitis.

Case: A 39-year-old female patient with abdominal pain was referred to our institution and was found to have a pelvic mass. After a thorough evaluation, surgical exploration revealed the presence of TOA. No evidence of gastrointestinal disease was present. The patient's history was significant for an uncomplicated total abdominal hysterectomy for benign disease of the uterus four years prior. Abscess cultures grew Streptococcus intermedius.

Conclusion: This case reports the rare occurrence of TOA in a patient who had undergone an abdominal hysterectomy four years prior to presentation. If the patient reports a surgical history of prior hysterectomy, TOA is often stricken from consideration. Although unlikely, adnexal abscess formation should be considered in the differential diagnosis of a patient with abdominal pain and a pelvic mass, even with a remote history of hysterectomy. Infect. Dis. Obstet. Gynecol. 6:138-140, 1998. () 1998 Wiley-Liss, Inc.
\end{abstract}

A cute pelvic inflammatory disease (PID) affects nearly 1 to $2 \%$ of young, sexually active women, accounting for approximately one million new cases per year in the United States. ${ }^{1}$ It is estimated that $10 \%$ of acute PID cases will be complicated by tubo-ovarian abscess (TOA) formation. Even with advances in antibiotic coverage and perioperative care, the mortality rate from ruptured TOA can approach $10 \%$.

The microorganisms most commonly associated with PID include Neisseria gonorrhoeae and Chlamydia trachomatis. However, TOAs are usually mixed infections and can culture both aerobic and anaerobic organisms. This etiology necessitates the use of broad-spectrum antibiotics for treatment of TOAs. Patients with these infections may report abdominal and/or pelvic pain and fever and have a concurrent pelvic mass.

Patients who have undergone hysterectomy are thought to be protected by the surgical interruption of the anatomic continuity between the upper and lower genital tracts. Furthermore, pelvic surgery itself is unlikely to be complicated with adnexal abscess formation. Ledger et al. ${ }^{2}$ reported only a $2.7 \%$ incidence of postoperative adnexal abscess formation in a series of 470 consecutive vaginal

*Correspondence to: Dr. Ira R. Horowitz, Emory University School of Medicine, Department of Gynecology and Obstetrics, 1639 Pierce Drive, Atlanta, GA. 30322 
hysterectomies. However, no abscesses were identified in a series of 819 abdominal hysterectomies by the same authors.

\section{CASE REPORT}

A 39-year-old woman, para 1001, arrived at an outlying institution reporting a one-week history of worsening lower abdominal pain. The pain was described as dull with occasional sharp exacerbations. The pain was not relieved by oral medications, nor was it related to position. The patient had not experienced nausea, vomiting, fevers, or other gastrointestinal complaints, nor did she have vaginal bleeding, discharge, or urinary symptoms.

Her medical history revealed no contributory information. Significant events in her surgical history were cesarean delivery in 1978, a bilateral tubal ligation in 1980, and a total abdominal hysterectomy for a leiomyomatous uterus four years prior to the current event. Postoperatively, she had not experienced any infection or fever.

A computed tomographic scan of the abdomen and pelvis performed prior to the patient's referral to our institution revealed bilateral complex pelvic masses and para-aortic lymphadenopathy. The patient was referred to our institution for evaluation and treatment for a suspected ovarian malignancy. Upon reevaluation, the patient reported persistent lower abdominal pain with no other associated signs or symptoms. Physical exam revealed a blood pressure of 100/70 $\mathrm{mmHg}$, pulse rate of $84 \mathrm{bpm}$, and an oral temperature of $38^{\circ} \mathrm{C}$. Abdominal examination was unremarkable except for lower abdominal tenderness without rebound tenderness or guarding. Pelvic exam was significant for a leftsided fixed mass, approximately six centimeters in size and tender to palpation. Rectal examination was confirmatory, and the stool was negative for occult blood.

Laboratory evaluation revealed the white blood cell count to be $17,400 / \mathrm{mL}$ with $2 \%$ bands and hematocrit of 36.8. Serum electrolytes and chemistries were within normal limits. Blood and urine cultures were without growth. A chest radiograph was negative except for some apical scarring. A contrast enhanced computed tomographic scan of the abdomen and pelvis was obtained revealing bilateral complex, predominantly cystic adnexal masses (Fig. 1), and multiple enlarged para-aortic

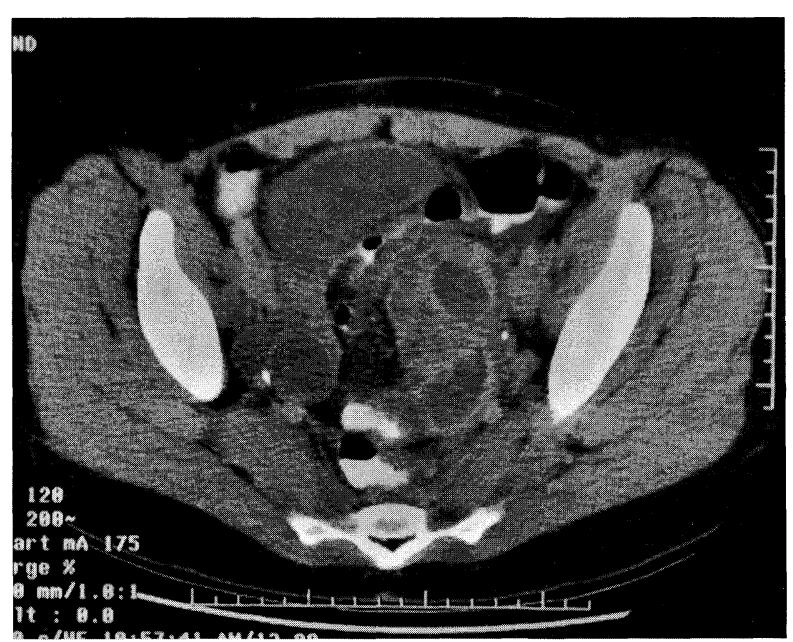

Fig. I. Computed tomographic scan of the pelvis showing complex pelvic masses.

lymph nodes. The serum CA125 level was within the normal range.

After a repeat temperature of $38^{\circ} \mathrm{C}$ was recorded, the patient was administered intravenous ticarcillin/clavulanic acid ( $3.1 \mathrm{~g}$ every 6 hours). The low-grade fever of $38^{\circ} \mathrm{C}$ persisted for the first three days of admission. On that day a repeat white blood cell count was $20,400 / \mathrm{mL}$, and the patient's symptoms were not improving. Her abdominal pain was persistent, and the finding of bilateral pelvic masses was worrisome. She was counseled about the possibility of malignancy versus an infectious process and consented to exploratory surgery.

Intraoperative findings included a $6 \times 6-\mathrm{cm}$ mass in the left adnexae adhered to the pelvic sidewall. This structure grossly appeared to be the patient's tube and ovary complexed in an inflammatory mass. In the right adnexae, a $3 \times 3-\mathrm{cm}$ tubo-ovarian complex was also noted. Frozen section analysis confirmed an inflammatory nature with no evidence of malignancy. Enlarged para-aortic lymph nodes were excised and revealed the presence of a reactive, inflammatory process with no evidence of malignancy or necrotic tumor. The appendix was excised and grossly normal. The bowel was run in its entirety with no evidence of abscess formation or diverticular disease. Cultures of the abscesses were taken at the time of surgery.

The patient had an uneventful postoperative course. Antibiotic coverage was broadened by administering ampicillin ( $2 \mathrm{~g}$ every 6 hours), gentamicin (100 mg every 8 hours), and clindamycin (900 mg every 8 hours) intravenously. The patient 
remained afebrile and was tolerating a regular diet by the third postoperative day, and she was discharged home with a prescription for two weeks of oral antibiotics.

At the follow-up visit four weeks after the surgery, the patient was doing well and had no complaints. Final pathologic analysis showed a normal appendix and reactive lymph nodes. The left adnexae contained acute salpingitis with complex TOA formation; the right adnexae was also a TOA with an incidental follicular cyst. Final blood and aerobic abscess cultures were negative; however, anaerobic abscess culture grew Streptococcus intermedius.

\section{DISCUSSION}

Adenexal abscesses are uncommon complications of pelvic surgery that may result from a variety of procedures, including cesarean delivery, dilation and curettage, tubal ligation, salpingo-oophorectomy, and abdominal hysterectomy. However, the most common procedure complicated by abscess formation is vaginal hysterectomy. In Ledger's study, $73 \%$ of abscesses occurred after vaginal hysterectomy, $16 \%$ after vaginal tubal ligation, and $11 \%$ after cesarean delivery.

Abscesses usually present late in the postoperative period. The time interval to development has ranged from a few days to one report of six years. ${ }^{3}$ Most cases seem to occur within a few weeks to several months. ${ }^{4,5}$ Only two cases occurring after abdominal hysterectomy are clearly described in the literature. Fletcher et al. $^{6}$ and Hueston ${ }^{3}$ have reported TOAs eight months and six years after abdominal hysterectomy, respectively. In both cases, as in this case, the source of the infection was unclear.

The accepted theory on PID-related TOA formation is that of an ascending infection via the uterus. However, in the absence of a uterus one can only speculate as to the cause of infection. Theories considered in this case included hematogenous seeding, although blood cultures were negative, and appendicitis. The patient denied a history of lower genital tract trauma, which could have initiated an ascending infection. In addition, there was no evidence of diverticular disease or vaginal fistula intraoperatively. Because the patient's postoperative course following hysterectomy was uncomplicated, it is also unlikely that the present infection originated at the time of her hysterectomy and was indolent. We tend to favor a combined transmural (gastrointestinal) and hematogenous seeding origin.

The microorganism isolated, $S$. intermedius, deserves mention. It is a member of the $S$. milleri group, which consist of $S$. intermedius, $S$. constellatus, and $S$. anginosus. These bacteria are strongly associated with the development of human abscesses. The $S$. milleri group is considered part of the normal human gastrointestinal and genitourinary flora. ${ }^{7}$ Interestingly, each is usually associated with a more predominant site of abscess formation. $S$. intermedius in particular is almost always isolated from pyogenic central nervous system or liver abscesses and is rarely, if ever, isolated from the genitourinary tract. ${ }^{7,8}$

This case report demonstrates the need to consider TOA in the differential diagnosis of a patient with a pelvic mass and abdominal pain, regardless of a history of prior hysterectomy. It also suggests that the current theory on TOA formation may only be a partial explanation of the pathophysiology of this complicated and life-threatening gynecologic condition. Added consideration must also be given to $S$. intermedius as a pathogen in TOA.

\section{REFERENCES}

1. Droegemueller W: Infections of the upper genital tract. In Mishell DR, Stenchever MA, Droegemueller W, Herbst AL (eds): Comprehensive Gynecology. St. Louis: Mosby, pp 661-690, 1997.

2. Ledger WJ, Campbell C, Taylor D: Adnexal abscess as a late complication of pelvic operations. Surg Gynecol Obstet 129:973-978, 1969.

3. Hueston WJ: A case of tubo-ovarian abscess 6 years after hysterectomy. J Ky Med Assoc 90:114-116, 1992.

4. Stone SC, LaRose PE: Tubo-ovarian abscess after vaginal hysterectomy. J La State Med Soc 131:241—243, 1979.

5. Livengood CH, Addison WA: Adnexal abscess as a delayed complication of vaginal hysterectomy. Am J Obstet Gynecol 143:596-597, 1982.

6. Fletcher JL, Nolan TE, Milam MJ: Late tubo-ovarian abscess following abdominal hysterectomy. J Fam Pract 33:190-192, 1991.

7. Whiley RA, Beighton D, Winstanley TG, Fraser HY, Hardie JM: Streptococcus intermedius, Streptococcus constellatus, and Streptococcus anginosus (the Streptococcus milleri group): association with different body sites and clinical infections. J Clin Microbiol 30:243-244, 1992.

8. Melo JC, Raff MJ: Brain abscess due to Streptococcus MG-intermedius (Streptococcus milleri). J Clin Microbiol 7:529-532, 1978. 


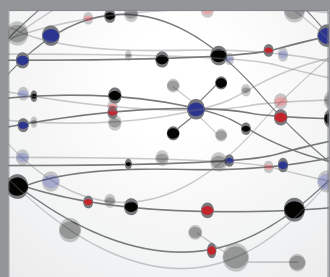

The Scientific World Journal
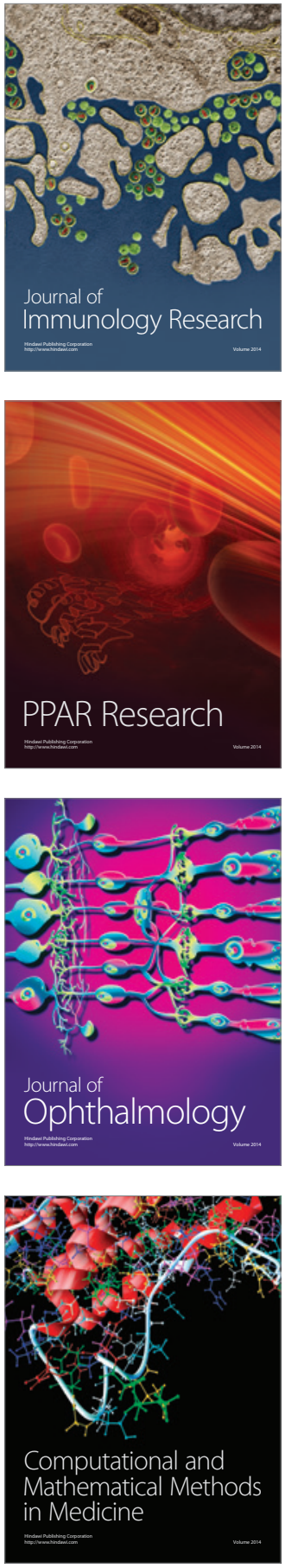

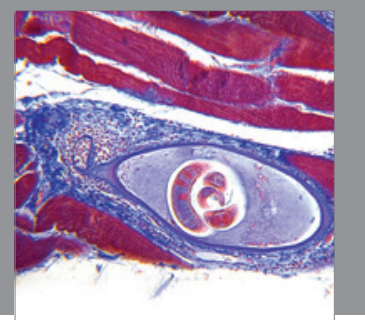

Gastroenterology

Research and Practice
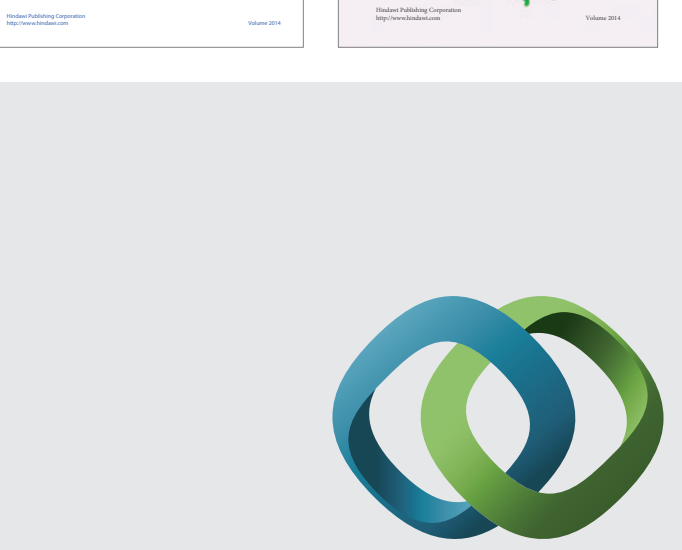

\section{Hindawi}

Submit your manuscripts at

http://www.hindawi.com
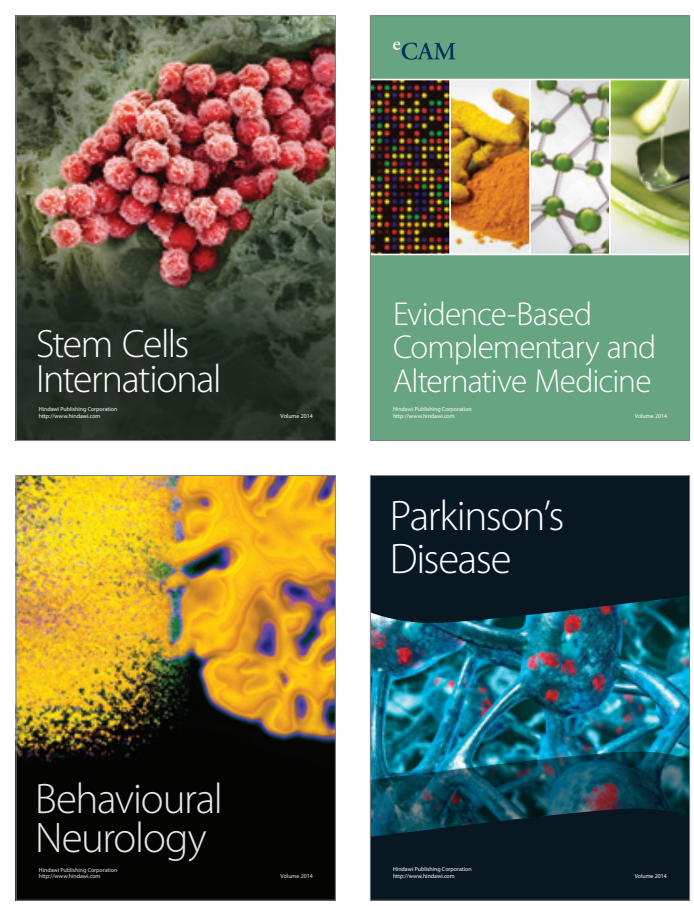

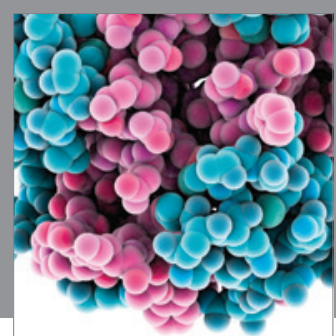

Journal of
Diabetes Research

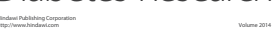

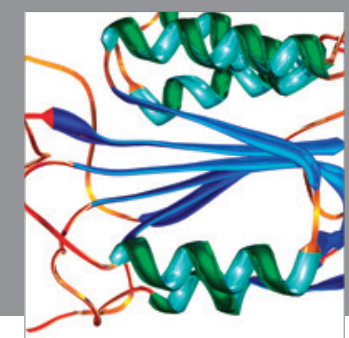

Disease Markers
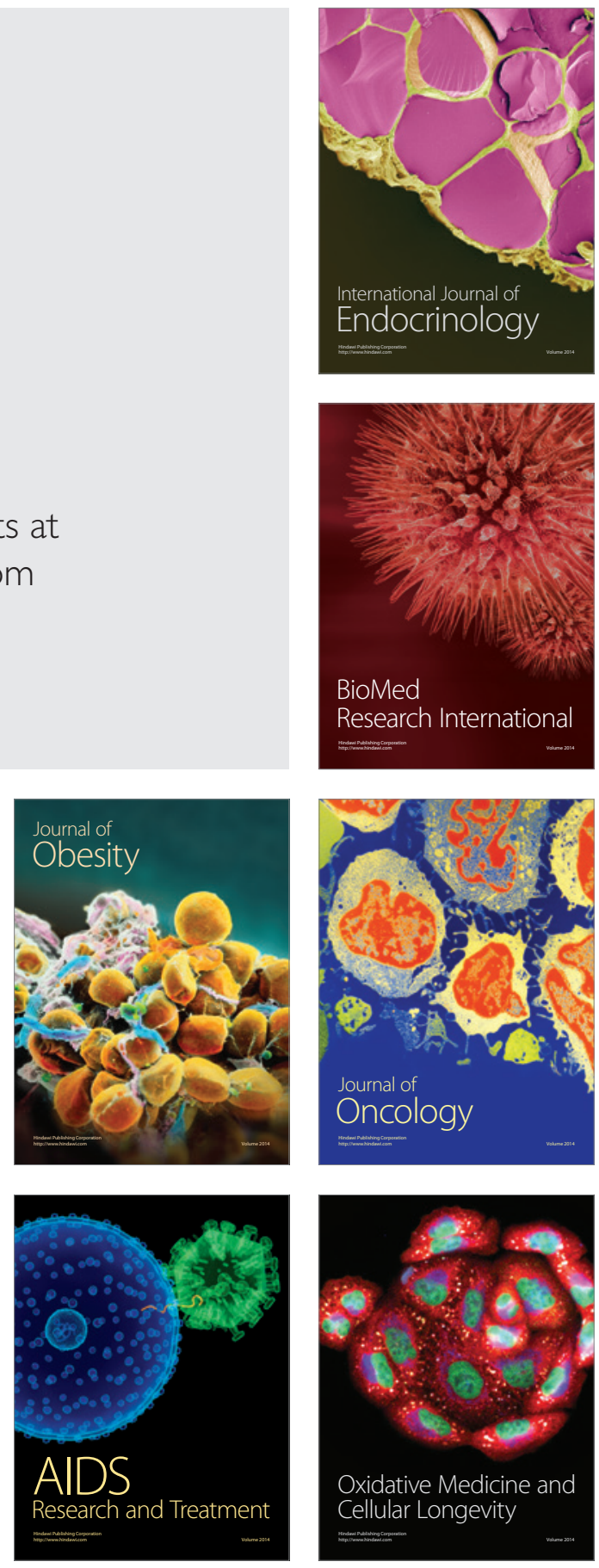